\title{
A Novel System for Cloud-Based Micro Additive Manufacturing of Metal Structures
}

\author{
Anne Brant ${ }^{1}$ and Murali M. Sundaram ${ }^{2}$ \\ ${ }^{1,2}$ Department of Mechanical and Materials Engineering, University of Cincinnati, Cincinnati, \\ USA \\ brantam@mail.uc.edu,murali.sundaram@uc.edu
}

\begin{abstract}
Cloud-based computing holds enormous potential for collaboration, cost-saving, streamlining, and versatility of manufacturing. Additive manufacturing, being a computer-based system that can save point-by-point data of parts to be manufactured, can be easily integrated into the cloud. Preliminary work was done to test the cloud-based application of an in-house micro metal additive manufacturing by electrochemical deposition process. The system was linked to commercial cloud and email access for constant real-time communication from any user with a phone, tablet, or personal computer. The process could be started, stopped, altered, and queried remotely via the cloud. Input parameters (ie: geometry, tool size) and preliminary design rules (ie: current feedback threshold value) were specified. Plots of output performance, time, and current information were communicated back to the user ondemand, as well as stored on the cloud long-term. The cloud could then link input parameters to the history of system performance on such input parameters in a cloud-stored database. An experiment was set up to optimize horizontal deposition parameters based on deposition resolution, and save these values into the cloud for future use, The experiment was successfully executed and demonstrates the advantage of long-term storage, knowledge sharing, and convenience that the cloud offers for the manufacturing realm.
\end{abstract}

Keywords: cloud-manufacturing, additive manufacturing, electrochemical deposition

\section{Introduction}

\subsection{Overview}

All areas of manufacturing are being drastically reshaped by new demands and possibilities brought on by globalization and increasing use of the Internet. In particular, cloud computing holds a great amount of potential to streamline the manufacturing process. Advantages include centralized data management, resource scaling, transparency and communication among business groups and clients, and flexibility in location of operation. The features and advantages of the cloud and its 
context in manufacturing are investigated in this introduction. This is then followed by the challenges and lack of experience that hinder widespread commercial adoption of the cloud, particularly for additive manufacturing at the small scale.

\subsection{Cloud-Based Computing}

According to the National Institute for Standards and Technology (NIST), cloud-based computing is a model for widespread, location-independent access to shared computing resources (Mell \& Grance, 2009). The "cloud" includes both the software and hardware resources that make this possible; consumption of these resources can be easily measured by the provider, allowing for allocation towards more heavily-used functions (Armbrust et al., 2010). Cloud computing usage can encompass several levels of control and access to specific individuals, specific groups, or the general public (Armbrust et al., 2010; Mell \& Grance, 2009). While specific boundaries are still being debated (Armbrust et al., 2010), cloud computing can be divided into levels of control: "Software as a service", the shared user access of provided software on the cloud; "Platform as a service", the ability for developers to upload and share their own software; and at the most fundamental level, "Infrastructure as a service", the fundamental level of control by administrators of processing, storage, networks, and operating systems (Mell \& Grance, 2009).

\subsection{Cloud-Based Manufacturing}

The emergence of the cloud is drastically reshaping the manufacturing industry. Examples include the remote control and monitoring of Computer Numerical Controlled (CNC) and robotic devices (L. Wang, Gao, \& Ragai, 2014), tracking of the material flow of a manufacturing process (Wu, Rosen, \& Schaefer, 2014b), feature recognition during mid-manufacture of a part (Anbalagan, Wang, \& Weidong, 2014), and usage of past data to create future manufacturing suggestions (Ren, Zhang, Hou, Wu, \& Teng, 2014). Cloud computing enables the "Design Anywhere, Manufacture Anywhere" (DAMA) philosophy to emerge. Design, manufacturing, management, testing, demand, and supply chain data can be shared transparently between manufacturers, wholesalers, and retailers. Travel can be avoided by allowing for virtual communication among individuals and machines. Timeconsuming searches can be avoided, because the cloud is able to automatically search for and suggest the necessary equipment, individuals, and knowledge needed to manufacture a product. Overall, the cloud-based manufacturing system encompasses the following services: design, manufacturing, experimentation, simulation, management, maintenance and information integration (Tao, Zhang, Venkatesh, Luo, \& Cheng, 2011). The cloud provides centralized management of all of these capabilities, and a high degree of customization for the user (Xu, 2012). Additionally, the cloud enables tracking any given product from beginning to the very end of life, even including the performance of the product in the hands of the end user. Such tracking is critical to the future product improvements (X. V. Wang \& Xu, 2014).

\subsection{Cloud-Based Additive Manufacturing}

Additive manufacturing is a natural candidate for cloud based manufacturing. For example, 3D Hubs in Europe allow customers to be linked to 3D printing services in their area. Additionally, researchers at Georgia Tech have worked on the DMCloud, which is a prototype cloud service with additive manufacturing capabilities. There are features that can identify materials and machines for a specific part, evaluate the manufacturability of a specific part by a specific machine, store design rules, and store training materials and wizards for users (Wu, Rosen, \& Schaefer, 2014a). Additive manufacturing also has inherent advantages in cloud integration over conventional manufacturing processes. A frequently-discussed advantage of cloud manufacturing is the automatic scaling of 
resources based on customer use. However, limitations in tooling supply for conventional processes can inhibit this and still require time to manufacture the correct tooling. Because many additive manufacturing process do not require tools, their resources can be seamlessly scaled by the cloud without such limitations (Wu, Thames, Rosen, \& Schaefer, 2013). Or, additive manufacturing can be used as a "rapid tooling" process to create conventional manufacturing tools on-demand (Rosen, Chen, Sambu, Allen, \& Mistree, 2003).

\subsection{Limitations}

While cloud-based manufacturing is redefining the manufacturing industry and has enormous potential, there are still limitations that need to be overcome. Bottlenecks to wider commercial adoption include: effective standards of use, reliable access, knowledge management, connection of the user to appropriate manufacturing resources, virtualization of machines, operational cost, energy usage, compatibility of older files with newer cloud software, and security of the stored data (Tao et al., 2011; Yang, Gao, Fan, Wang, \& Wang, 2014). There also remains much work to be done to adopt cloud computing and additive manufacturing as a commercial technique, particularly with additive manufacturing techniques on the smaller scale. This paper aims to provide preliminary work into the usage of cloud resources for micro additive manufacturing of metal parts using an electrochemical deposition process.

\section{Experimental Setup}

\subsection{Physical Setup}

An in-house electrochemical setup developed in prior work (Brant, Sundaram, \& Kamaraj, 2015; Sundaram, Kamaraj, \& Kumar, 2015) was used to perform micro additive manufacturing. The setup is illustrated in Figure 1(a). Stepper motor signals and tool voltage were applied to the system, and toolsubstrate current monitored by measuring the voltage across a $100 \Omega$ resistor $R$ connected in series. The controller was connected to the cloud and ran a mixture of commercial and open-source software to communicate with the cloud. As seen in Figure 1(b), forms were manufactured by moving the substrate relative to the tool during deposition, corresponding to a generated tool path. Current feedback was taken at regular current-checking points along the tool path. The current was monitored at each point until it exceeded a threshold of $50 \mathrm{~mA}$ or a constant time elapsed. The system would then be prompted to move the substrate so that the tool would reach the next current-checking point along the tool path. 


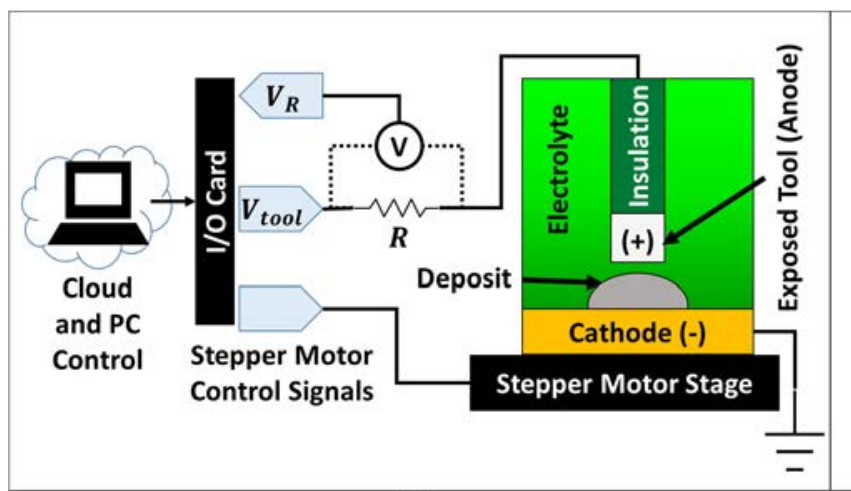

(a)

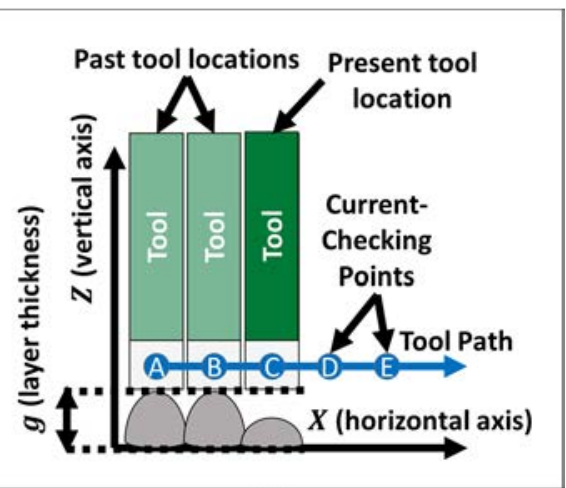

(b)

Figure 1. (a) Experimental setup and connections and (b) generation of a part by simultaneous stepper motor movement, deposition, and current feedback

\subsection{Tool Path and Current Feedback}

Figure 2 summarizes the link between physical tool behavior, voltage across the resistor, and the manufacturing instructions. When dealing with an isolated point, Figure 2(a) and Figure 2(b) show that a low voltage $V_{R}$ is measured across the resistor $R$ if there is no metal deposited beneath the tool, or if the deposited metal has not yet covered the tool-substrate gap. Figure 2(c) shows that a voltage spike occurs when the deposit touches the tool. Figure 2(d) and Figure 2(e) show that when dealing with a chain of multiple points to deposit, the tool can hook onto or otherwise maintain contact with a previously-deposited point, thus giving a false deposition reading. Because there is a short circuit, no current passes through the electrolyte and the next point is prevented from being deposited. In the current-feedback method, the system is instructed to move the stepper motors after a voltage spike is sensed, and to maintain its current position if not sensed. The voltage spike corresponds to a current through the resistor above the threshold value. The current through the resistor can be calculated from $V_{R}$ using Ohm's law. Figure 2(f) shows a solution to these issues by deliberately depositing the metal lower than the tool-substrate gap. Because the system cannot monitor an intermediate current between the low current and spike, this can be achieved by implementing an optimal constant deposition time for each point, independent of current feedback. 


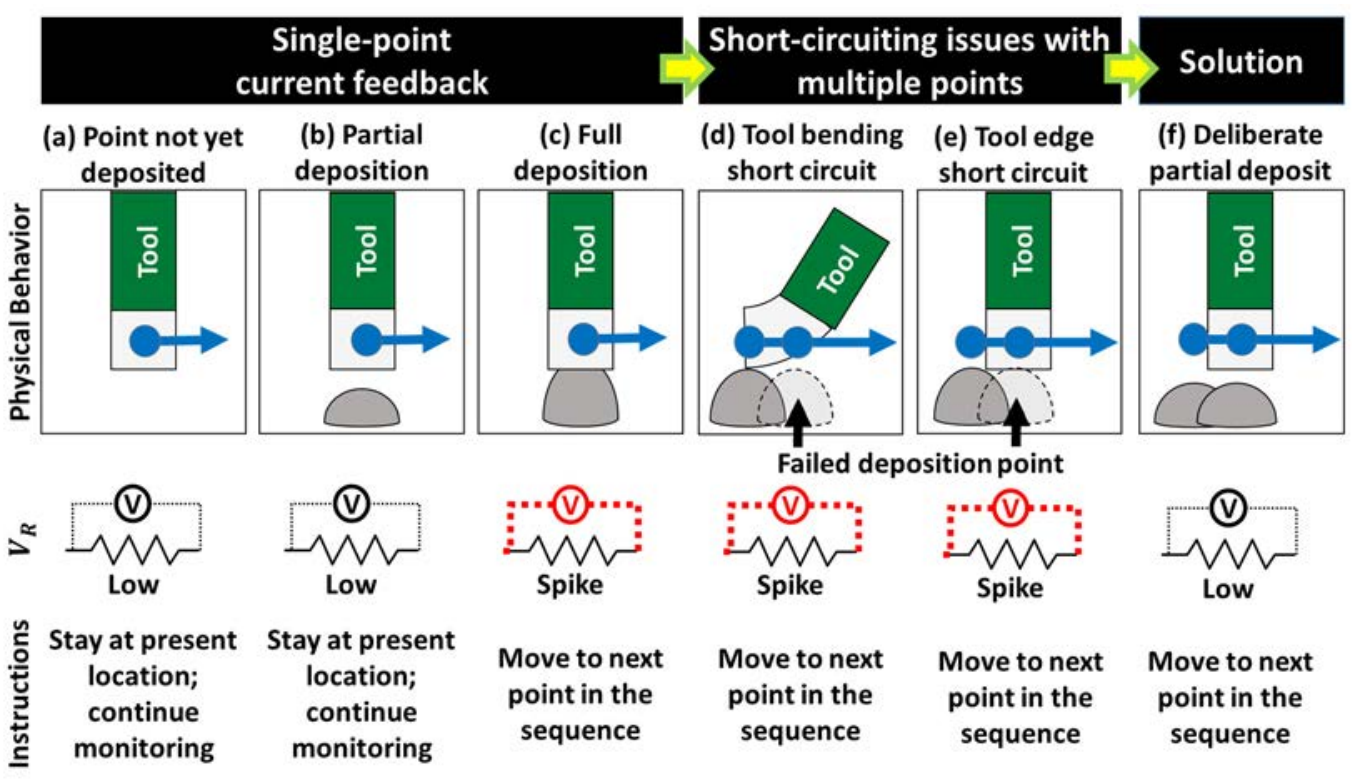

Figure 2. Link between physical behavior, current feedback, and manufacturing instructions

\subsection{Cloud System Overview}

A prototype system was constructed by integrating the in-house electrochemical additive manufacturing system with cloud capability, as summarized in Figure 3. Specifically, the "Platform as a service" capability of the cloud was explored, with custom algorithms uploaded to the cloud. The code was sent via the cloud to the controller, which physically controlled an in-house additive manufacturing setup. The following capabilities were explored:

a) Generate manufacturing instructions for a three dimensional part as input into the cloud.

b) Control the manufacturing process from any location via the cloud, including starting and stopping the process on-demand, as well as modifying parameters within the setup such as motor speed, motor direction, and voltage application, via the cloud.

c) Provide on-demand feedback across the cloud on the real-time performance of the setup.

d) Provide suggestions by the cloud for setup change based on the measured feedback. 


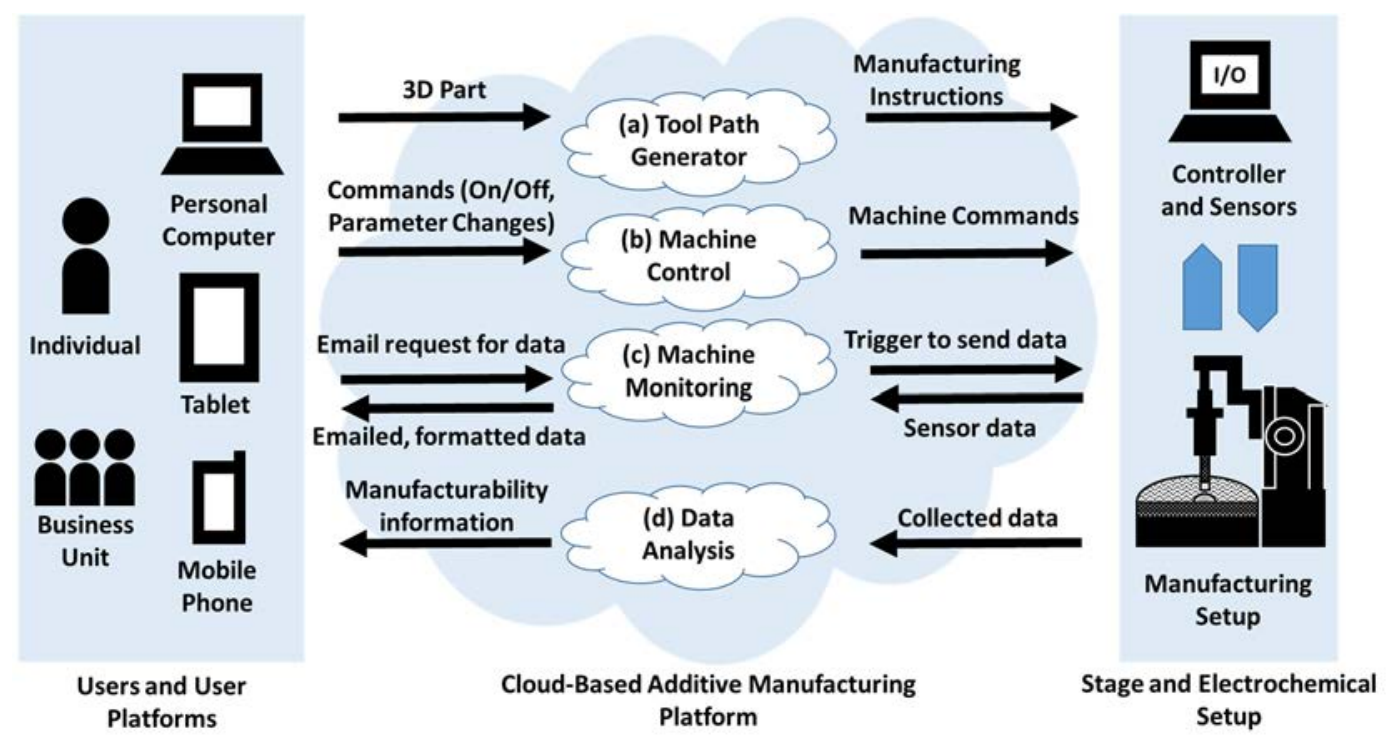

Figure 3. User and manufacturing setup interaction via cloud-based features

\subsection{Manufacturing Instructions}

For this work, the form of a cylinder was used as an input and the tool path was generated. The user inputs are summarized in Table 1. Note that the tool path generation properties (Layer Thickness and Horizontal Fineness) are expressed in discrete motor steps, which are equivalent to a linear displacement of $1.5 \mu \mathrm{m}$.

Table 1. Input parameters

\begin{tabular}{ccc}
\hline Scope & Parameters & Value \\
\hline Manufacturing & Tool Diameter $\left(D_{T}\right)$ & $100 \mu \mathrm{m}$ \\
Setup & Default Current Threshold $\left(I_{t}\right)$ & $30 \mathrm{~mA}$ \\
\hline Geometric Part & Cylinder Height $(H)$ & $500 \mu \mathrm{m}$ \\
Input & Cylinder Diameter $(D)$ & $500 \mu \mathrm{m}$ \\
& Wall Thickness $(T)$ & $D_{T}$ \\
\hline Tool Path & Layer Thickness $(L)$ & $10 \mathrm{steps}$ \\
Generation & Horizontal Fineness $(F)$ & 1 step \\
\hline
\end{tabular}

\subsection{On-Demand Control}

As shown in Figure 3(see (b) Machine Control), the system was set up to monitor for incoming messages from the user in the form of email. The emails provided the following instructions:

- What part to manufacture and its manufacturing instructions

- Commands to start, pause, or terminate the manufacturing process of a given part

- Commands to manually control stage position and applied tool voltage

- Commands to change process parameters mid-manufacture

When such an email was detected, the system would send commands to the controller to perform the requested tasks. Some were completely physical, such as stage position, tool voltage, and start/stop 
instructions. Others were software-based, such as the manufacturing instructions, or current threshold used by the controller.

\subsection{On-Demand Feedback}

As summarized in Figure 3(see (c) Machine Monitoring), the user could request information about the manufacturing process via an email command from any location. Devices compatible with email, such as a tablet, computer, or phone, could be used. The system was set up to email a spreadsheet and plot including information about the current point, and overall metrics of the process up until the present point, as detailed in Table 2 .

Table 2. On-demand feedback sent to cloud

\begin{tabular}{|c|c|}
\hline Current Point Information & Overall Metrics \\
\hline $\begin{array}{l}\text { - Index of point currently deposited } \\
\text { - The time elapsed since initialization } \\
\text { - Visual plot of deposition } \\
\text { - If current point is in a chain of } \\
\text { "sticking points" }\end{array}$ & $\begin{array}{l}\text { - Maximum point deposition time } \\
\text { - The maximum length of "sticking } \\
\text { points" } \\
\text { - Deposition time plot for all points } \\
\text { Mean, variance, and standard deviation } \\
\text { of deposition times }\end{array}$ \\
\hline
\end{tabular}

Based on the feedback on the currently-manufactured point, the user could be aware of the status of the overall manufacturing process, and information about the tool behavior at each point. An issue was experimentally observed, for many points, where the tool would remain electrically connected to a given point, and therefore be inhibited from depositing the next points in the correct manner. The deposition time information helped give insight into when this was happening, and the data was used in the next experiment to solve this issue using a smart manufacturing technique. This strategy is detailed in 2.7 .

\subsection{Smart Manufacturing}

The Smart Manufacturing algorithm was used to determine the optimal constant deposition time for each point in order to deposit each point to partial height as illustrated in Figure 2(f), in order to avoid short-circuiting and false deposition readings illustrated in Figure 2(d) and Figure 2(e).

The deposition times vary based upon geometrical and electrical parameters (Brant et al., 2015), which can be varied infinitely. A cloud database that can store the ideal point deposition time for a given set of process parameters is an extremely useful tool that can save the time, effort, and cost required to figure it out on the spot by individual users. The ideal deposition time for gap sizes $2 \mu \mathrm{m}$ and $5 \mu \mathrm{m}$ was identified in the following manner, and then saved into the cloud for future trials where particles would be spaced apart at this amount.

1. Deposit a "pillar" of 50 points spaced vertically by gap size $g$, using current spike feedback.

2. Record the individual time of deposition for each point each pillar trial.

3. Repeat three pillar trials and collect statistics for deposition times (average and maximum).

4. Deposit a 5 point wide, 10 layer tall "wall", illustrated in Figure 4, using a fixed deposition time. When starting a new layer, ensure that the vertical gap is held constant by touching the tool down and raising the tool to the specified gap. Begin at an integer time interval in seconds 
that is nearest to the maximum deposition time for pillar trials of the gap. Perform three trials for any fixed time interval.

5. Repeat the constant time depositions, incrementing down in .10 second intervals. Record the tool-substrate current for each point before depositing, and any instant current spikes that occur. An instant spike current above $50 \mathrm{~mA}$ is defined as a "sticking point" indicating that the tool is short-circuited to the previous point and is not going to properly deposit at the next point.

6. Repeat iterations until a threshold deposition time value is reached, which separates a trial from absence or presence of "sticking" points.
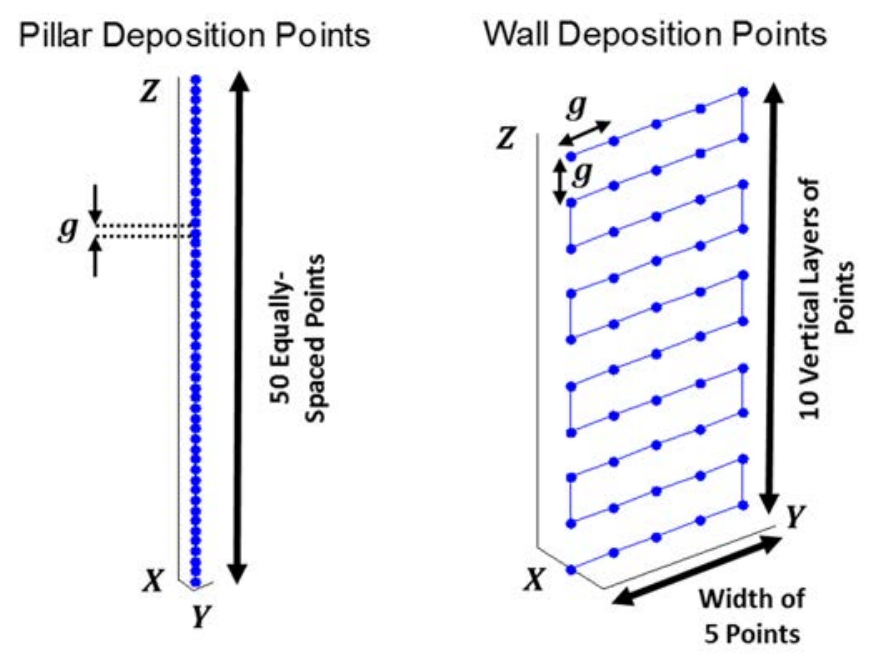

Figure 4. "Pillar" and "Wall" depositions used to gather data for smart manufacturing applications. The pillar represents vertical deposition properties and the wall investigates horizontal deposition optimization

\section{Results And Discussion}

\subsection{Manufacturing Instructions}

The tool path and current checking points were successfully generated for the cylinder. Figure 5 illustrates these results with additional close-ups for clarity.

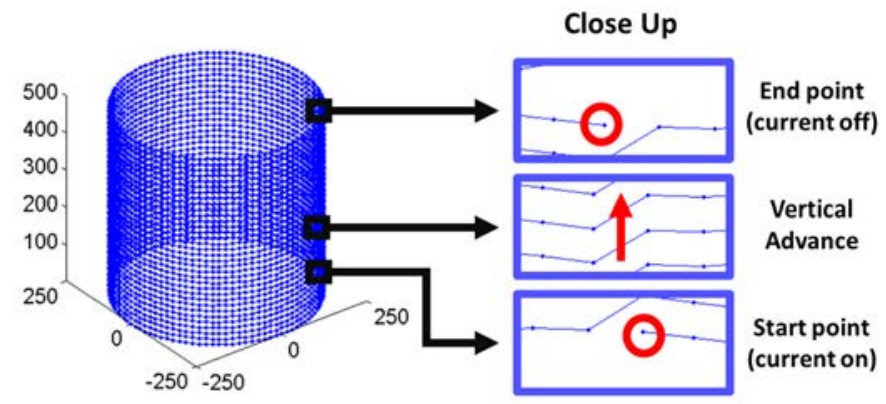

Figure 5. Generated tool path for cylinder 


\subsection{On-Demand Control}

The process was initialized via mobile phone by sending an email to the system. The software on the controller was able to reliably scan the cloud for incoming messages and receive the command, and start the program. Further on-demand control was then executed after receiving on-demand feedback, as detailed in section 3.3.

\subsection{On-Demand Feedback}

Feedback information, in the format detailed in section 2.6, was requested on-demand, at two arbitrary times, from a remote location. Figure 6 shows the information in the form of mobile phone screenshots.

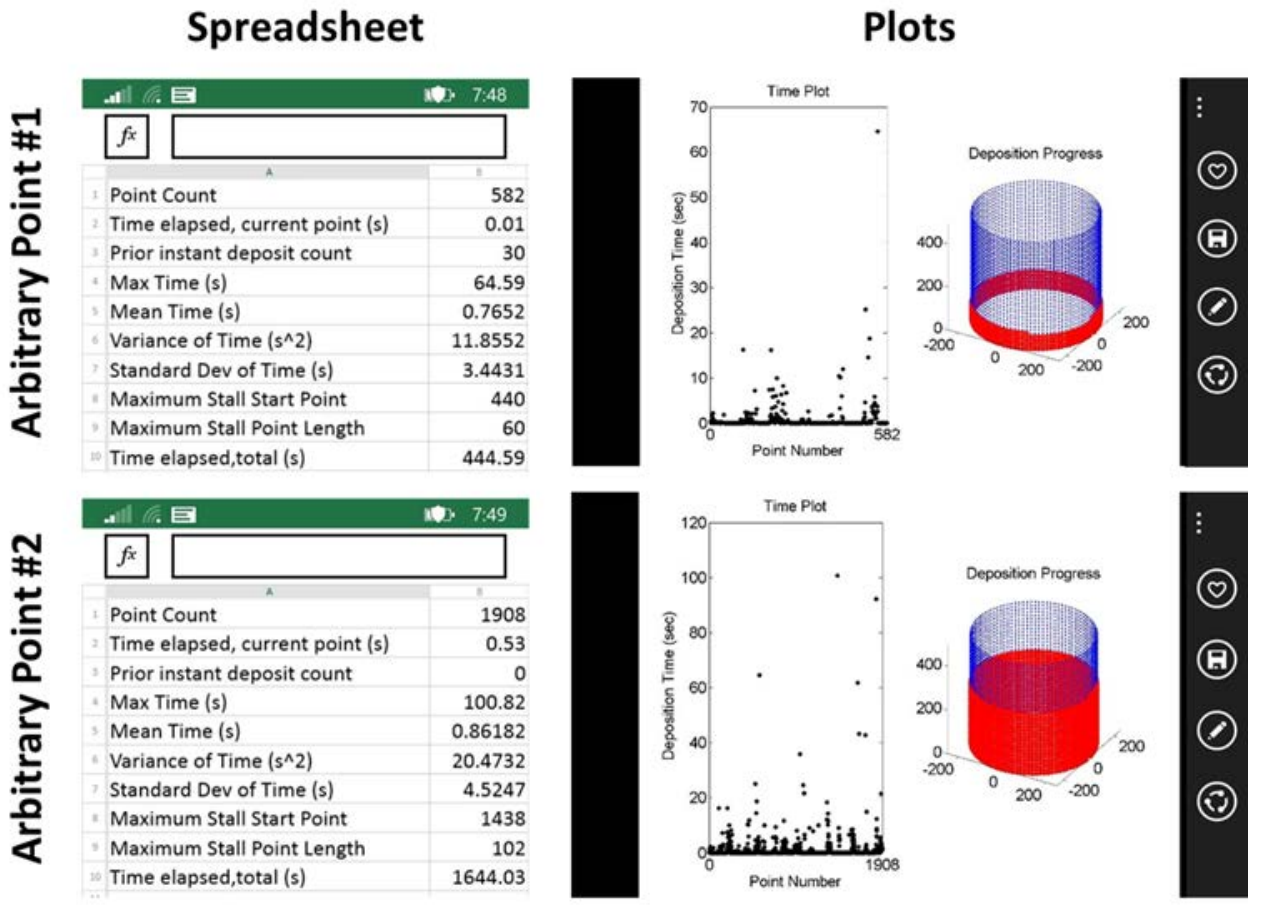

Figure 6. Mobile phone screenshots of cloud-based output from manufacturing setup

As seen in the plot for "Arbitrary Point \#1", there was seen to be a long chain of "tool sticking" points. At these points, a command was sent to the system to move the tool up one step at a time, until the short circuit was broken, in order to unstick the tool and continue manufacture. However, the integrity of the part was lost, due to the extra vertical movement and missed points while the tool was sticking. As seen in, this resulted in an imperfect, jagged form to be deposited instead of a cylinder.. Another issue seen in depositing the cylinder involved the bending or dislocation of the part away from the tool, resulting in the tool losing its location and being far away from the intended point to be deposited. This was evidenced by an overly-high elapsed time for the deposition of the current point so far. Due to the dislocation of the manufactured part, the only solution to this problem would be to restart the part entirely, with changed input parameters. In this case, the "Smart Manufacturing" algorithm, detailed in the next section, was designed in order to avert the short circuiting issues entirely. 


\subsection{Smart Manufacturing}

Two pillar calibration trials were performed at 2-step and 5-step gaps, and maximum point deposition times were obtained. Rounded up to the nearest .5 seconds, these times were 1 second and 2.5 seconds, respectively. Three trials of constant-time deposition of the "wall" shape were performed in decreasing .10 second increments. The number of tool sticking points, which had an initial measured current above $50 \mathrm{~mA}$, were counted for each trial and averaged for each time value. The results are shown in Figure 7.

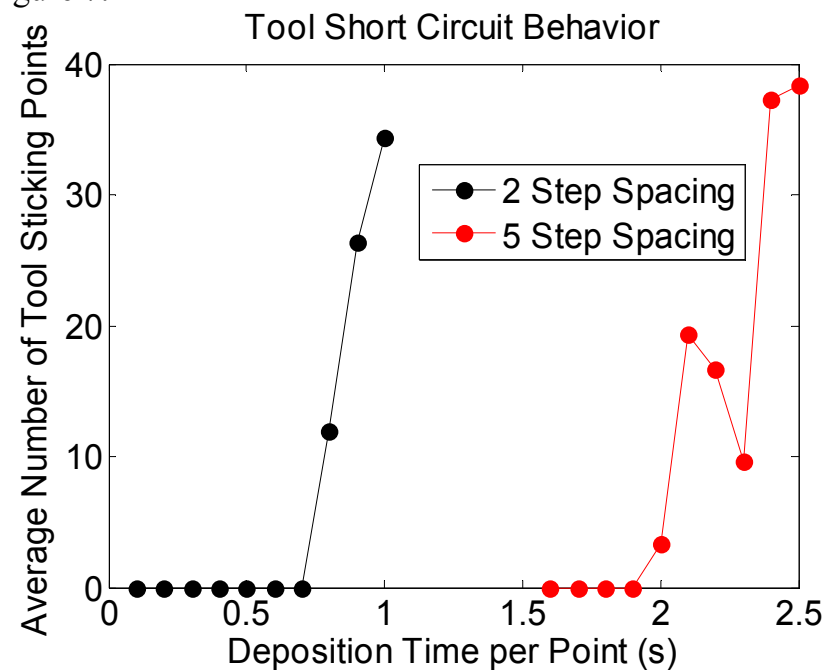

Figure 7. Characterization of tool short circuit behavior vs. deposition time allocated per point

As seen in the plot, the maximum deposition time thresholds that could be applied to avoid the deposit touching the tool for a structure with 2 steps and 5 steps between deposition points were found to be .70 and 1.90 seconds, respectively. It is also seen that the optimal time for a point to be deposited in a horizontal structure with no short circuiting is greater than the mean time for a point to deposit in a vertical pillar structure. This can be explained by current density behavior. For points in the pillar, the deposition is only taking place atop an area of one previously-deposited point, leading to a higher current density focused on a smaller area and faster deposition. However, during horizontal shape deposition, the tool is more exposed to surrounding substrate features on the same horizontal plane, leading to slower deposition. The relationship between vertical and horizontal deposition speed can then be evaluated, as seen in Table 3. The ratio of the horizontal optimal time to the vertical mean time can also be found and used as a characteristic parameter to describe the vertical vs. horizontal behavior being discussed. This parameter can then be readily saved into the cloud for prediction of what constant time is expected to deposit a complex, horizontally-layered shape if the average time of deposition of a pillar with the same gaps under current feedback is known.

This time data was used to recreate the cylinder of the same dimensions, of 500 step diameter and 500 step height, using optimal times found for the 2- and 5-step gap trials. The parameters are summarized in Table 3. As seen in the table, because the total cylinder height was held constant while the layer gap size was varied, the numbers of layers and points in the instructions differed. The effectiveness of the previously-found data was evaluated in its application to cylinder construction, and the resulting deposits are shown in Figure $8 \mathrm{~b}$ and Figure 8c. 
Table 3. Comparison of vertical and horizontal deposition behavior, generated experimental parameters for constant-time deposition, and total deposition times for constant-time method

\begin{tabular}{ccccccc}
\hline $\begin{array}{c}\text { Gap } \\
\text { Size } \\
\text { (steps) }\end{array}$ & $\begin{array}{c}\text { Mean } \\
\text { Vertical } \\
\text { Deposition } \\
\text { Time for } \\
\text { Pillar (s) }\end{array}$ & $\begin{array}{c}\text { Optimal } \\
\text { Deposition } \\
\text { Time for } \\
\text { Horizontal } \\
\text { Line (s) }\end{array}$ & $\begin{array}{c}\text { Ratio of } \\
\text { Horizontal } \\
\text { to Vertical } \\
\text { Time }\end{array}$ & $\begin{array}{c}\text { Layers } \\
\text { Required } \\
\text { to Build } \\
\text { 500-Step } \\
\text { Height } \\
\text { Cylinder }\end{array}$ & $\begin{array}{c}\text { Points } \\
\text { Required } \\
\text { to Build } \\
\text { 500-Step } \\
\text { Height } \\
\text { Cylinder }\end{array}$ & $\begin{array}{c}\text { Total } \\
\text { Deposition } \\
\text { Time (h) }\end{array}$ \\
\hline 2 & $\begin{array}{c}.38 \\
\text { trial 4) } \\
.89\end{array}$ & $.70 \mathrm{~s}$ & 1.58 & 250 & 25100 & 4.8 \\
$($ trial 8$)$ & $1.90 \mathrm{~s}$ & 2.13 & 100 & 10100 & 5.3 \\
\hline
\end{tabular}

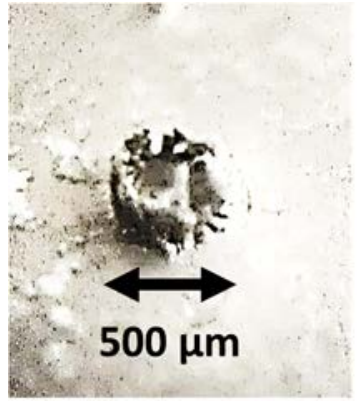

(a)

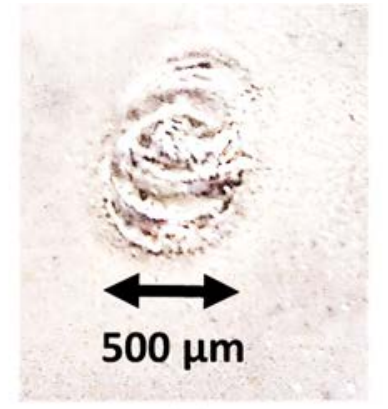

(b)

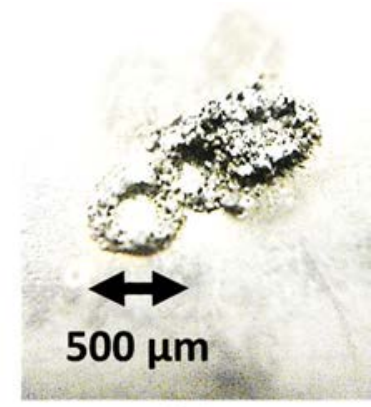

(c)

Figure 8. Optical micrographs of deposited shapes: (a) current-feedback preliminary deposition, (b) 2step gap with constant $.7 \mathrm{~s}$ deposition time, and (c) 5-step gap with constant $1.9 \mathrm{~s}$ deposition time

As seen in the deposition images, the tall, jagged spires that appeared in the current feedback deposition trial were not generated in the constant-time deposits. However, the layers were deposited in a horizontally-displaced position, resulting in a lower deposition height than intended. This can be explained by "sticking points" occurring due to the unevenness of the deposited part, leading to tool drift. The surface roughness for all depositions was evaluated by performing five arbitrarily-located 3 $\mu \mathrm{m}$ by $3 \mu \mathrm{m}$ atomic force microscope scans on each sample. The results for each deposition are summarized and compared side-by-side in Table 4.

Table 4. Surface roughness measurements of all deposits

\begin{tabular}{cccc}
\hline & $\begin{array}{c}\text { Current Feedback- } \\
\text { Based Deposit } \\
\text { Roughness (nm) }\end{array}$ & $\begin{array}{c}\text { 2-Step Layer, .7s } \\
\text { Constant Time Deposit } \\
\text { Roughness (nm) }\end{array}$ & $\begin{array}{c}\text { 5-Step Later, 1.9s } \\
\text { Constant Time } \\
\text { Deposit Roughness } \\
\text { (nm) }\end{array}$ \\
\hline Scan 1 & 58.832 & 120.35 & 55.694 \\
Scan 2 & 32.467 & 240.63 & 167.2 \\
Scan 3 & 87.250 & 94.226 & 87.838 \\
Scan 4 & 44.022 & 101.710 & 327.68 \\
Scan 5 & 14.817 & 45.313 & 250.730 \\
Average Roughness & 47.4776 & 120.4458 & 177.8284 \\
Range of Roughness & 72.433 & 195.317 & 271.986 \\
\hline
\end{tabular}


As seen in the average roughness values, the constant-time depositions yielded a higher average roughness, as well as a wider range of roughness values. While neither method produced an ideal cylinder, this data can be used by the cloud for surface roughness prediction for future scans, and help in the optimization process of micro electrochemical additive manufacturing.

\section{Conclusions}

The capabilities of the cloud for micro additive manufacturing applications were investigated. The potential for Smart Manufacturing applied to micro additive manufacturing was shown by saving experimental data of a current-feedback experiment and using it to generate a constant-time experiment. Both techniques were seen to yield imperfect deposits, but provide preliminary insight into cloud control of the process. Future work is required to optimize the techniques, or try a new technique entirely. Additionally, the alteration of tool size needs to be explored experimentally; this has been shown in simulations to have an impact on the electrical behavior and deposition geometry (Brant et al., 2015).

Overall, using the cloud for manufacturing applications opens up a multitude of unprecedented possibilities for the manufacturing realm, particularly in micro and nano manufacturing, where much data is still being collected. A collective cloud storage allows for worldwide sharing of specific information and codes for micro and nano processes to accelerate progress. Future work involves the application of the cloud-based system to other types of additive manufacturing processes and conventional manufacturing processes. This would require adapting the cloud and sensing systems to the unique feedback inherent in the different types of processes. This allows both additive and traditional manufacturing to attain the benefits of cloud-based control.

\section{Acknowledgements}

Financial support provided by the National Science Foundation under Grant No CMMI-1400800 is acknowledged.

\section{References}

Anbalagan, A., Wang, S., \& Weidong, L. A Cloud Based Feature Recognition System to Support Collaborative and Adaptive Process Planning. In: Proc. of the ASME 2014 International Manufacturing Science and Engineering Conference collocated with the JSME 2014 International Conference on Materials and Processing and the 42nd North American Manufacturing Research Conference 2014, pp. V001T004A024-V001T004A024.

Armbrust, M., Fox, A., Griffith, R., Joseph, A. D., Katz, R., Konwinski, A., . . . Stoica, I. A view of cloud computing. Communications of the ACM 2010; 53(4): 50-58.

Brant, A. M., Sundaram, M. M., \& Kamaraj, A. B. Finite Element Simulation of Localized Electrochemical Deposition for Maskless Electrochemical Additive Manufacturing. Journal of Manufacturing Science and Engineering 2015; 137(1): 011018.

Mell, P., \& Grance, T. The NIST definition of cloud computing. National Institute of Standards and Technology 2009; 53(6): 50.

Ren, L., Zhang, L., Hou, B., Wu, Q., \& Teng, D. Intelligent User Interface in Cloud Manufacturing. In: Proc. of the ASME 2014 International Manufacturing Science and Engineering 
Conference collocated with the JSME 2014 International Conference on Materials and Processing and the 42nd North American Manufacturing Research Conference 2014, pp. V001T004A025-V001T004A025.

Rosen, D. W., Chen, Y., Sambu, S., Allen, J. K., \& Mistree, F. The rapid tooling testbed: a distributed design - for - manufacturing system. Rapid Prototyping Journal 2003; 9(3): 122-132.

Sundaram, M. M., Kamaraj, A. B., \& Kumar, V. S. Mask-Less Electrochemical Additive Manufacturing: A Feasibility Study. Journal of Manufacturing Science and Engineering 2015; 137(2): 021006.

Tao, F., Zhang, L., Venkatesh, V. C., Luo, Y., \& Cheng, Y. Cloud manufacturing: a computing and service-oriented manufacturing model. In: Proc. of the Institution of Mechanical Engineers, Part B 2011, pp. 0954405411405575.

Wang, L., Gao, R., \& Ragai, I. An Integrated Cyber-Physical System for Cloud Manufacturing. In: Proc. of the ASME 2014 International Manufacturing Science and Engineering Conference collocated with the JSME 2014 International Conference on Materials and Processing and the 42nd North American Manufacturing Research Conference 2014, pp. V001T004A029V001T004A029.

Wang, X. V., \& Xu, X. Cloud Manufacturing in Support of Sustainability. In: Proc. of the ASME 2014 International Manufacturing Science and Engineering Conference collocated with the JSME 2014 International Conference on Materials and Processing and the 42nd North American Manufacturing Research Conference 2014, pp. V001T004A014-V001T004A014.

Wu, D., Rosen, D. W., \& Schaefer, D. Cloud-based design and manufacturing: status and promise. Springer, 2014a.

Wu, D., Rosen, D. W., \& Schaefer, D. Modeling and analyzing the material flow of crowdsourcing processes in cloud-based manufacturing systems using stochastic petri nets. In: Proc. of the ASME 2014 International Manufacturing Science and Engineering Conference collocated with the JSME 2014 International Conference on Materials and Processing and the 42nd North American Manufacturing Research Conference 2014b, pp. V001T004A011V001T004A011.

Wu, D., Thames, J. L., Rosen, D. W., \& Schaefer, D. Enhancing the Product Realization Process With Cloud-Based Design and Manufacturing Systems. Journal of Computing and Information Science in Engineering 2013; 13(4): 041004.

$\mathrm{Xu}, \mathrm{X}$. From cloud computing to cloud manufacturing. Robotics and computer-integrated manufacturing 2012; 28(1): 75-86.

Yang, Y., Gao, R. X., Fan, Z., Wang, J., \& Wang, L. Cloud-Based Prognosis: Perspective and Challenge. In: Proc. of the ASME 2014 International Manufacturing Science and Engineering Conference collocated with the JSME 2014 International Conference on Materials and Processing and the 42nd North American Manufacturing Research Conference 2014, pp. V001T004A028-V001T004A028. 